\title{
The impact of a Housing First randomized controlled trial on substance use problems among homeless individuals with mental illness
}

\author{
Maritt Kirst, Suzanne Zerger, Vachan Misir, Stephen Hwang \& \\ Vicky Stergiopoulos
}

\begin{abstract}
Version Post-Print/Accepted Manuscripts
Citation Kirst, M., Zerger, S., Misir, V., Hwang, S., \& Stergiopoulos, V. (2015).

(published version) The impact of a Housing First randomized controlled trial on substance use problems among homeless individuals with mental illness. Drug and Alcohol Dependence 146, Pages 24-29.

Copyright / License

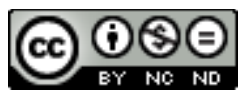

This work is licensed under a Creative Commons AttributionNonCommercial-NoDerivatives 4.0 International License.
\end{abstract}

Publisher's Statement The final published version of this article is available at Elsevier via https://dx.doi.org/10.1016/j.drugalcdep.2014.10.019.

Always cite the published version, so the author(s) will receive recognition through services that track citation counts, e.g. Scopus. If you need to cite the page number of the TSpace version (original manuscript or accepted manuscript) because you cannot access the published version, then cite the TSpace version in addition to the published version using the permanent URI (handle) found on the record page. 
The impact of a Housing First randomized controlled trial on substance use problems among homeless individuals with mental illness

Maritt Kirst ${ }^{1,2^{*}}$, Suzanne Zerger ${ }^{1}$, Vachan Misir ${ }^{1}$, Stephen Hwang ${ }^{1,3}$, \& Vicky Stergiopoulos ${ }^{1,4}$

1 Centre for Research on Inner City Health, St. Michael's Hospital, 30 Bond St., Toronto, ON M5B 1W8

2 Toronto Central Community Care Access Centre, 250 Dundas St. W., Toronto ON M5T 2Z5

3 Department of Medicine, University of Toronto, 200 Elizabeth St., Toronto ON M5G 2C4

4 Department of Psychiatry, University of Toronto, 250 College St., 8th floor, Toronto, ON M5T 1R8

*Corresponding author present address:

Toronto Central Community Care Access Centre

250 Dundas St. West

Toronto ON M5T 2ZS

Telephone: 416-217-3820 ext. 2551

Email: maritt.kirst@utoronto.ca 


\begin{abstract}
Background: There is strong evidence that Housing First interventions are effective in improving housing stability and quality of life among homeless people with mental illness and addictions. However, there is very little evidence on the effectiveness of Housing First in improving substance use-related outcomes in this population. This study uses a randomized control design to examine the effects of scatter-site Housing First on substance use outcomes in a large urban centre.
\end{abstract}

Methods: Substance use outcomes were compared between a Housing First intervention and Treatment as Usual group in a sample of 575 individuals experiencing homelessness and mental illness, with or without a co-occurring substance use problem, in the At Home/Chez Soi trial in Toronto, Canada. Generalized linear models were used to compare study arms with respect to change in substance use outcomes over time (baseline, 6, 12, 18 and 24 month).

Results: At 24 months, participants in the Housing First intervention had significantly greater reductions in number of days experiencing alcohol problems and amount of money spent on alcohol than participants in the Treatment as Usual group. No differences between the study arms in illicit drug outcomes were found at 24 months.

Conclusions: These findings show that a Housing First intervention can contribute to reductions in alcohol problems over time. However, the lack of effect of the intervention on illicit drug problems suggests that individuals experiencing homelessness, mental illness and drug problems may need additional supports to reduce use.

Trial Registration: Current Controlled Trials ISRCTN42520374

Key words: homelessness; substance use; mental illness; Housing First 


\section{Introduction}

The prevalence of homelessness in many Canadian cities continues to rise, despite the development of services targeting this issue. Research has shown that the prevalence of mental illness and addictions is higher among homeless individuals than in the general population, and that homeless individuals often have complex unmet service needs (Fischer \& Breakey, 1986; Goering et al., 2011; D. Padgett, Struening, \& Andrews, 1990). Studies have found that $25-70 \%$ of homeless individuals have cooccurring mental health and substance use problems (Collins et al., 2012; Drake, Osher, \& Wallach, 1991; D. K. Padgett, Gulcur, \& Tsemberis, 2006; Palepu, Patterson, Moniruzzaman, Frankish, \& Somers, 2013; Street Health, 2007), and co-occurring substance use presents a considerable hindrance to mental health recovery (D. K. Padgett, Stanhope, Henwood, \& Stefancic, 2011). This subpopulation of homeless individuals is particularly vulnerable, and is more likely to experience chronic physical illness, premature death, longer length of time homeless, and poor treatment retention (Palepu, Patterson, Strehlau, \& al., 2013).

\subsection{Housing First}

Housing First (HF) is an intervention designed to address the unique needs of this subpopulation; it provides permanent housing without prerequisites for abstinence or treatment, and access to supportive health services. This approach contrasts with more traditional supportive housing models that require sobriety and engagement in mental health treatment before consumers are deemed "housing ready"(Tsemberis, Gulcur, \& Nakae, 2004). Operating from a harm reduction philosophy, the Housing First approach posits that providing housing to homeless individuals with mental illness first provides the foundation, stability and safety necessary for consumers to move towards recovery (Tsemberis et al., 2004). Several research studies have supported its designation as an "evidence-based practice," showing consistently positive outcomes on residential stability, reductions in cost of public services, and improved quality of life (D. K. Padgett et al., 2006; Pearson, Montgomery, \& Locke, 2009; Perlman \& Parvinsky, 2006; Tsai, Mares, \& Rosenheck, 2010; Tsemberis et al., 2004). Researchers continue to caution, however, that variation in HF programs, lack of consistent fidelity measures, and methodological constraints weaken the current knowledge base, and recommend more research seeking clarity about its effectiveness for specific subpopulations (Groton, 2013; Kertesz, Crouch, Milby, Cusimano, \& Schumacher, 2009; Mark, 2014).

In particular, there is no consistent evidence regarding the effectiveness of HF for individuals with active substance use problems. Authors reviewing comparative trials involving HF concluded that "for homeless individuals with a prominent and active problem of addiction, the data on Housing First are 
mixed and unsettled," arguing that most of the program studies have served individuals experiencing chronic homelessness but whose "severity of substance misuse" has been moderate." (Kertesz et al., 2009). Variability in measurements of substance use contributes to mixed findings, though it is widely acknowledged that assessing substance use with dually diagnosed or homeless persons is especially complicated (Sacks, Drake, Williams, Banks, \& Herrell, 2003).

Though one of the most consistent outcomes has been HF's positive impact on housing stability and retention, varied effects of substance use on mediating that outcome emerge in the research literature. One study, for example, found consumers without a substance use diagnosis were much more likely to achieve consistent stable housing than those with a diagnosis, especially those with both alcohol and drug problems (Hurlburt, Hough, \& Wood, 1996). Other studies have similarly echoed the impact of substance use on predicting shorter tenure in housing (Collins, Malone, \& Clifasefi, 2013; Lipton, Siegel, Hannigan, Samuels, \& Baker, 2000; Wong et al., 2006), though others taking substance use into account have found significant differences in housing outcomes (Edens, Mares, Tsai, \& Rosenheck, 2011; Palepu, Patterson, Moniruzzaman, et al., 2013; Pearson et al., 2009).

Effects of HF on substance use outcomes remain unclear. One recent review of the five most rigorous HF studies concluded that the majority found neither HF nor the control group programs decreased substance use (Groton, 2013), affirming the same conclusion from an earlier review (Kertesz et al., 2009). A recent study using interactional analysis suggested these effects "may not be universal across subgroups;" these authors found, for example, that African American veterans in the HF program had greater reductions in severity of drug problems than Caucasians (O'Connell, Kasprow, \& Rosenheck, 2012). Another study assessing differences in substance use outcomes between Housing First and Treatment First (i.e., temporary congregate housing with prerequisite of detoxification/sobriety and 'housing readiness') participants using qualitative data, found that participants who received Treatment First were more likely to use drugs and/or abuse alcohol 12 months after program entry than Housing First participants (Padgett et al. 2011). Studies based on an HF program in Seattle serving chronically homeless persons with severe alcohol problems found steady decreases in daily alcohol use, and reductions in median number of drinks and number of days intoxicated among the intervention group (Collins et al., 2012; Larimer et al., 2009).

\subsection{The At Home/Chez Soi Project}

In 2009, the Mental Health Commission of Canada initiated the At Home/Chez Soi Project, a multi-site randomized controlled trial to assess the effectiveness of scatter-site Housing First in the Canadian context (Goering et al., 2011; Hwang, Stergiopoulos, O'Campo, \& Gozdzik, 2012). The mixed (quantitative and qualitative) methods study followed participants for two years post enrollment. The At 
Home/Chez Soi project was implemented across five cities in Canada - Moncton, Montreal, Toronto, Winnipeg and Vancouver. Inclusion criteria for the study were: aged 18 or older; absolutely homeless or precariously housed status; the presence of a mental health disorder with or without a co-occurring substance use disorder, as determined by the Mini International Neuropsychiatric Interview (MINI 6.0) (Goering et al., 2011). At Home/Chez Soi project participants were randomized to either scatter-site Housing First or 'Treatment as Usual' (TAU). Participants in the Housing First intervention received choice in the location of housing across the city, a rent supplement, and mental health service supports according to their level of need. In the Toronto site, 97 participants were randomized to an intervention designed for those with high service needs (housing + Assertive Community Treatment), 204 were randomized to a moderate needs intervention (housing + Intensive Case Management), and 274 were randomized to the TAU arm. Those randomized to the TAU arm received no specialized services but received information materials about services available in the community. Moderate needs participants who identified as ethnoracial were provided with the option to participate in an Ethnoracial-ICM intervention (ER-ICM - specific to the Toronto site of the project). A description of the study intervention arms has been published elsewhere (Goering et al., 2011). Study participants were classified as high or moderate need using criteria that correspond to Section 3 of the Ontario Standards for Assertive Community Treatment teams (Goering et al., 2011). All other study participants were classified as moderate need.

This study examines the effect of HF on substance use outcomes by comparing scatter- site HF and treatment as usual for a population of homeless adults with mental illness in Toronto, Canada. We examined the impact of the HF intervention on both alcohol and drug use problems, and addressed the following research question: are there differences in substance use (alcohol and illicit drug) outcomes between the Housing First intervention group and TAU group over time? We hypothesized that participants in the HF intervention arms would have greater reductions in substance use problems over time than participants in the TAU group.

\section{Materials and Methods}

\subsection{Data Collection}

Participants in both study arms completed surveys at baseline, and at 3 month intervals up until 24 months after enrollment to assess changes by study arm in such outcomes as mental health, substance use, social functioning, community integration, and criminal justice system involvement. Participants were also asked about sociodemographic characteristics at baseline. Interviews were conducted by trained interviewers in the project office or in participants' homes. Interviews were conducted between 2009 and 2013. Written consent was received from all study participants. The follow-up rate for the sample at 24 
months was $80 \%$. Ethics approval was received for the study from the St. Michael's Hospital Research Ethics Board.

\subsection{Study Measures}

2.2.1.Outcome Variables: Substance use problem outcome variables included the Global Assessment of Individual Need - Substance Dependence Scale Short Screener (GAIN-SS) (Dennis, Chan, \& Funk, 2006), and four questions derived from the Addiction Severity Index (ASI) (McLellan et al., 1992). The GAIN-SS consisted of five questions to determine participants' severity of substance use problems (such as getting into fights, problems at work, dealing with withdrawal symptoms ) in the 'past month', '2-12 Months' or ' 1 or more years.' Using these questions, the GAIN Past Month score is calculated by counting the number of times the participant identified that they had these problems in the last month. The resulting score has a range from 0 to 5 with higher scores reflecting greater severity of substance use problems (Dennis et al., 2006). Individual ASI items included the number of days in the past month that alcohol problems and drug problems (defined as craving, withdrawal symptoms, disturbing effects of use, or wanting to stop and being unable to) were experienced and the amount of money spent on alcohol and drugs in the last 30 days (McLellan et al., 1992). The ASI items on the number of days in the past month that alcohol or drug problems were experienced ranged from 0-30. The ASI questions on the amount of money spent on alcohol involved a response range of $\$ 0-\$ 900$, and the amount spent on drugs involved a range of $\$ 0-\$ 10,000$ in the last 30 days.

2.2.2. Covariates: Covariates included study treatment, a dichotomous variable indicating randomization to the Housing First interventions (ACT, ICM and ER-ICM) or Treatment as Usual. Time reflects the five survey data points (baseline, 6 months, 12 months, 18 months and 24 months). The effects of a time by study treatment interaction were also included to examine changes in substance use outcomes by study arm over time. Potential confounder variables added to the models included: gender, age at enrolment, visible minority status, country of birth, marital status, duration of homelessness, employment, education, chronic conditions, and number of children, as these have been examined as confounders in other studies of the effects of Housing First on substance use problems (Edens et al., 2011; D. K. Padgett et al., 2011).

\subsection{Data Analysis}

A repeated measures negative binomial (generalized estimating equations) model was used for all count outcomes, (GAIN-SS Past Month Score, and number of days experiencing alcohol and drug problems), to account for the correlation between measures over time as well as to account for any overdispersion in 
our data, as opposed to a poisson model which is commonly used for count outcomes. For the continuous outcomes of amount of money spent on drugs and alcohol, a linear repeated measures (generalized linear model) model was used.

To determine the effect of each potential confounder on the main effect of treatment, time and their interaction, each variable was solely entered into the model to determine their effect including any two or three-way interactions with the intervention and time. Variables found to have a significant effect at a pvalue of $<0.2$ were then selected for a final multivariate model. All variables found to be significant at this stage were entered into a multivariate model and examined at a p-value of $<0.05$. Variables found to be non-significant in this model were further removed taking into account any confounding effects and collinear variables.

In the multivariate models, the incidence rate ratios are presented for each of the count outcomes which represent the change in count for each covariate with respect to the reference group or a unit change for continuous covariates (age). A significant change from 1 is indicative of an increase or decrease in the number of days substance use problems were experienced. Parameter estimates ( $\beta \mathrm{s})$ are presented for the continuous outcomes of amount of money spent on alcohol and drugs, with a positive or negative coefficient being indicative of an increase or decrease in the amount of money spent. A treatment by time interaction was included in all models in order to display the effect of the intervention over time with respect to each outcome, whereas interactions tested for each separate covariate were found to be nonsignificant (at a p-value of 0.05) and were further removed. Therefore, the IRRs and parameter estimates for each covariate represents an overall change across all time points.

All analysis was performed in SAS 9.4 using PROC GENMOD (count) and PROC MIXED (linear) for each outcome with an unstructured correlation matrix considered in all models.

\section{Results}

Table 1 shows sociodemographic characteristics for the entire sample and a comparison by study arm. The majority of the sample was male (68\%), and the mean participant age at enrollment was $39(\mathrm{SD}=12)$. The only significant difference between participants in the intervention arms and TAU was age at enrolment - TAU participants were slightly older than intervention participants (41 vs. 39 years old; pvalue $=0.0480$ ).

Table 2 displays substance use patterns at baseline for the total sample and by study arm. Of the 575 participants, $50 \%$ reported using alcohol, $44 \%$ reported using at least one illicit substance and $31 \%$ reported using more than one substance per day including alcohol, in the last 30 days. The only difference 
in substance use patterns between the intervention and TAU groups was that more TAU participants used opiates $(6 \%$ vs. $12 \%$; p-value $=0.016)$ and cocaine $(4 \%$ vs. $9 \%$; $p$-value $=0.011)$ in the last 30 days.

Table 3 presents results from the adjusted multivariate models for the four substance use problem outcomes. A declining trend in overall substance use problem symptoms (GAIN-SS) over time was observed with a significant reduction at 24 months (IRR=0.80; CI: 0.66, 0.97; $\mathrm{p}<0.05$ ). A significant treatment by time interaction was noted, with the intervention group having experienced fewer substance use problem symptoms at 12 months (IRR=0.74; CI: 0.58, 0.94; $\mathrm{p}<0.05$ ) than TAU participants, but not at 24 months. For the number of days participants experienced alcohol problems, a similar trend in reduction over time was observed, as the intervention group had experienced fewer days of problems at 6 (IRR=0.57; CI: 0.33, 0.99; p<0.05) 12 (IRR=0.54; CI: 0.30, 0.98; p<0.05) and 24 months (IRR=0.46; CI: $0.23,0.91 ; \mathrm{p}<0.05)$ than TAU participants. Participants in the intervention group also experienced a decline in the amount of money spent on alcohol, with a significant decrease at 24 Months $(\beta=-73.36$; CI: $-136.58,-10.14 ; \mathrm{p}<0.05)$, compared to TAU participants. The models for drug use problems and the amount of money spent on drugs did not show significant interactions for the intervention over time, but a general decrease among participants in the amount of money spent on drugs was observed at 18 months $(\beta=-102.13 ; \mathrm{CI}:-199.34,-4.92 ; \mathrm{p}<0.05)$.

\section{Discussion}

Study findings show that Housing First participants experienced greater reductions in alcohol problems over time than TAU participants. The study thus addresses an ongoing debate as to whether Housing First can have a positive impact on clinical outcomes such as substance use, in addition to improving housing stability and quality of life. These findings add to results from other studies that have found that the intervention contributes to reduced alcohol problem symptoms over time (Collins et al., 2012; Larimer et al., 2009; Tsemberis, Kent, \& Respress, 2012), but not necessarily reductions in illicit drug problems (D. K. Padgett et al., 2006; Tsai et al., 2010). It is not clear why a significant difference in substance use problem symptoms (GAIN-SS score) between HF and TAU groups was observed at 12 months and not sustained at 24 months, but future studies should examine the consistency of this pattern.

A number of studies have found that substance users enrolled in Housing First can achieve long term housing stability despite active substance use (Edens et al., 2011; Mares \& Rosenheck, 2011; D. K. Padgett et al., 2006; D. K. Padgett et al., 2011; Palepu, Patterson, Moniruzzaman, et al., 2013). Nevertheless, the lack of effect of the intervention on illicit drug use problems may be because individuals experiencing these problems need additional supports to reduce use. 
Some limitations to the analyses should be considered when interpreting results. Self -report measures of substance use were used, which could reflect under-reporting by participants. However, there is increasing evidence that self-reports of substance use can be valid and accurate among this population (D. K. Padgett et al., 2006). The use of the ASI questions of amount of money spent on alcohol or drugs as independent indicators of substance use and related problems is a limitation as participants could have received alcohol or drugs without necessarily spending money for substances.

Strengths of the study include the use of standardized measures of substance use problems. Furthermore, the analyses include an examination of intervention effects over time for alcohol and illicit drug problems separately - a number of studies in the Housing First research have examined one of these outcomes or combined them. The differential effects of Housing First on alcohol and illicit drug problems found in this study highlight that more research is needed on the specific role and efficacy of alcohol and illicit drug use treatment components, and suggest that specific patterns and types of use need to be considered in the delivery of Housing First interventions. 
References

Collins, S. E., Malone, D. K., \& Clifasefi, S. L. (2013). Housing retention in single-site Housing First for chronically homeless individuals with severe alcohol problems. American Journal of Public Health, 103(Supplement 2, No. S2), S269-S274.

Collins, S. E., Malone, D. K., Clifasefi, S. L., Ginzler, J., Garner, M., Burlingham, B., . . Larimer, M. E. (2012). Project-Based Housing First for Chronically Homeless Individuals With Alcohol Problems: Within-Subjects Analyses of 2-Year Alcohol Trajectories. American Journal of Public Health, 102(3), 511-519.

Dennis, M. L., Chan, Y.-F., \& Funk, R. R. (2006). Development and validation of the GAIN short screener (GSS) for internalizing, externalizing and substance use disorders and crim/violence problems amon adolescents and adults. The American Journal on Addictions, 15(S1), 80-91.

Drake, R. E., Osher, F. C., \& Wallach, M. A. (1991). Homelessness and dual diagnosis. American Psychologist, 36, 1149-1158.

Edens, E. L., Mares, A. S., Tsai, J., \& Rosenheck, R. A. (2011). Does active substance use at housing entry impair outcomes in supported housing for chronically homeless persons? Psychiatric Services, 62(2), 171-178.

Fischer, P. J., \& Breakey, W. R. (1986). Homelessness and mental health: An overview. International Journal of Mental Health, 14(4), 6-41.

Goering, P. N., Streiner, D. L., Adair, C., Aubry, T., Barker, J., Distasio, J., . . . Zabkiewicz, D. M. (2011). The At Home/Chez Soi trial protocol: a pragmatic, multi-site, randomised controlled trial of a Housing First intervention for homeless individuals with mental illness in five Canadian cities. BMJ Open, 1(2), e000323. doi: 10.1136/bmjopen-2011-000323

Groton, D. (2013). Are Housing First programs effective? A research note. Journal of Sociology \& Social Welfare, 40(1), 51.

Hurlburt, M., Hough, R., \& Wood, P. (1996). Effects of substance abuse on housing stability of homeless mentally ill persons in supported housing. Psychiatric Services, 47(7), 731-736.

Hwang, S., Stergiopoulos, V., O'Campo, P., \& Gozdzik, A. (2012). Ending homelessness among people with mental illness: The At Home/Chez Soi randomized trial of a Housing First intervention in Toronto. BMC Public Health, 12, 787.

Kertesz, S., Crouch, K., Milby, J. B., Cusimano, R. E., \& Schumacher, J. E. (2009). Housing First for Homeless Persons with Active Addiction: Are We Overreaching? Milbank Quarterly, 87(2), 495534.

Larimer, M. E., Malone, D. K., Garner, M. D., Atkins, D. C., Burlinham, B., Lonczak, H. S., . . Marlatt, G. A. (2009). Health care and public service use and costs before and after provision of housing for chronically homeless persons with severe alcohol problems. Journal of the American Medical Association (JAMA), 301(13), 1349-1357.

Lipton, F. R., Siegel, C., Hannigan, A., Samuels, J., \& Baker, S. (2000). Tenure in Supportive Housing for Homeless Persons with Severe Mental Illness. Psychiatric Services, 51(4), 479-486.

Mares, A. S., \& Rosenheck, R. A. (2011). A comparison of treatment outcomes among chronically homeless adults receiving comprehensive housing and healthcare services versus local care. Administration and Policy in Mental Health, 38.

Mark, M. (2014). Housing Programs Effective, but Studies Plagued by Limitations. Psychiatric News, 49(6), 9.

McLellan, A. T., Kushner, H., Metzger, D., Peters, R., Smith, I., Grissom, G., . . Argeriou, M. (1992). The fifth edition of the Addiction Severity Index. Journal of Substance Abuse Treatment, 9(3), 199213. 
O'Connell, M. J., Kasprow, W. J., \& Rosenheck, R. A. (2012). Differential impact of supported housing on selected subgroups of homeless veterans with substance abuse histories. Psychiatric Services, 63(12), 1195-1205.

Padgett, D., Struening, E. L., \& Andrews, H. (1990). Factors affecting the use of medical, mental health, alcohol and drug treatment services by homeless adults. Medical Care, 28(9), 805-821.

Padgett, D. K., Gulcur, L., \& Tsemberis, S. (2006). Housing First services for people who are homeless with co-occurring serious mental illness and substance abuse. Research on Social Work Practice, 16(1), 74-83.

Padgett, D. K., Stanhope, V., Henwood, B. F., \& Stefancic, A. (2011). Substance use outcomes among homeless clients with serious mental illness: Comparing Housing First with Treatment First programs. Community Mental Health Journal, 47(2), 227-232.

Palepu, A., Patterson, M., Strehlau, V., \& al., e. (2013). Daily substance use and mental health symptoms among a cohort of homeless adults in Vancouver, British Columbia. Journal of Urban Health, 90(4), 740-746.

Palepu, A., Patterson, M. L., Moniruzzaman, A., Frankish, C. J., \& Somers, J. (2013). Housing First improves residential stability in homeless adults with concurrent substance dependence and mental disorders. American Journal of Public Health.

Pearson, C., Montgomery, A. E., \& Locke, G. (2009). Housing stability among homeless individuals with serious mental illness participating in Housing First programs. Journal of Community Psychology, 37(3), 404-417.

Perlman, J., \& Parvinsky, J. (2006). Denver Housing First collaborative: Cost benefit analysis and program outcomes report. Denver: Colorado Coalition for the Homeless.

Sacks, J. A. Y., Drake, R. E., Williams, V. F., Banks, S. M., \& Herrell, J. M. (2003). Utility of the time-line follow-back to assess substance use among homeless adults. The Journal of Nervous and Mental Disease, 191(3), 145-153.

Street Health. (2007). The Street Health Report. Toronto: Street Health.

Tsai, J., Mares, A. S., \& Rosenheck, R. A. (2010). A multisite comparison of supported housing for chronically homeless adults: 'Housing First' versus 'Residential Treatment First'. Psychological Services, 7(4), 219-232.

Tsemberis, S., Gulcur, L., \& Nakae, M. (2004). Housing First, consumer choice, and harm reduction for homeless individuals with dual diagnosis. American Journal of Public Health, 94(4), 651-656.

Tsemberis, S., Kent, D., \& Respress, C. (2012). Housing stability and recovery among chronically homeless persons with co-occurring disorders in Washington, DC. American Journal of Public Health, 102(1), 13-16.

Wong, Y.-L. I., Hadley, T. R., Culhane, D. P., Poulin, S. R., Davis, M. R., Cirksey, B. A., \& Brown, J. L. (2006). Predicting staying in or leaving permanent supportive housing that serves homeless people with serious mental illness. Retrieved from http://shnny.org/uploads/Staying in or Leaving.pdf. 